\title{
AIAA-2002-1375 \\ Photogrammetry Methodology Development for Gossamer Spacecraft Structures
}

Richard S. Pappa and Thomas W. Jones

NASA Langley Research Center

Hampton, VA

Jonathan T. Black

George Washington University

Hampton, VA

Alan Walford

Eos Systems, Inc.

Vancouver, Canada

Stuart Robson

University College London

London, United Kingdom

Mark R. Shortis

University of Melbourne

Parkville, Australia

\section{3rd AIAA/ASME/ASCE/AHSIASC Structures, Structural Dynamics, and Materials Conference 3rd AIAA Gossamer Spacecraft Forum 22-25 April 2002 Denver, Colorado}





\section{Photogrammetry Methodology Development for Gossamer Spacecraft Structures}

\author{
Richard S. Pappa \\ NASA Langley Research Center \\ Hampton, VA 23681
}

\author{
Alan Walford \\ Eos Systems, Inc. \\ Vancouver, Canada
}

\author{
Thomas W. Jones \\ NASA Langley Research Center \\ Hampton, VA 23681 \\ Stuart Robson \\ University College London \\ London, United Kingdom
}

\author{
Jonathan T. Black \\ George Washington University \\ Hampton, VA 23681 \\ Mark R. Shortis \\ University of Melbourne \\ Parkville. Australia
}

\begin{abstract}
ABSTRAC $\mathbf{T}$
Photogrammetry--the science of calculating $3 \mathrm{D}$ object coordinates from images- is a flexible and robust approach for measuring the static and dynamic characteristics of future ultra-liglitweight and inflatable space structures (a.k.a., Gossanier structures), such as large membrane reflectors, solar sails, and thin-film solar arrays. Shape and dynanic measurements are required to validate new structut al modeling techniques and corresponding analytical models for these unconventional systems. Thi, paper summarizes experiences at NASA Langley Research Center over the past three years to develop or adapt photogrammetry methods for the specific problem of measuring Gossamer space structures. Turnkey industrial photogrammetry systems were not considered a cost-effective choice for thi: basic research effort because of their high purchase and maintenance costs. Instead, this research uses mainly off-the-shelf digitalcamera and software technologiw that are affordable to most organizations and provide acceptable accuracy.
\end{abstract}

\section{INTRODUCTION}

Photogrammetry is the science of measuring the size and location of 3D objects wising photographs. 'The classical application (known as topographic

Copyright 2002 by the American Institute of Aeronautics and Astronautics. Inc. No copyright is assen:d in the United States under Title 17. U.S. Code. The U.S. Government has a rovalty-free license to exercise all rights under the coysright claimed herein for Governmental Purposes. All other rights are reserved by the copyright owner. photogrammetry) is for creating aerial land surveys and maps. There are also many ground-based applications (known as non-topographic or close-range photogrammetry) in such diverse fields as archaeology, bioengineering, civil engineering, computer animation. forensic analysis, historical preservation, mechanical inspection, plant engineering, ship construction, and surgery. $^{2}$ Modern close-range photogrammetry uses digital imaging sensors ${ }^{3}$ and computer data analysis and often measures hundreds or thousands of object points. The fundamental theory is based on surveying principles." When dealing with time sequences of images, the term "videogrammetry" or "videometrics" is used to describe this technology." Photogrammetry offers the simplicity of taking photographs coupled with good to excellent measurement precision.

New analytical and experimental methods for shape and dynamic characterization of future Gossamer space structures, such as large membrane reflectors, solar sails, and thin-film solar arrays, are being developed at the NASA Langley Research Center (LaRC) and elsewhere." Accurate analytical methods are required for confident design of new or evolved structural concepts and for mission simulations. Correspondingly, experimental methods are required for measuring the shape and dynamic characteristics of research test articles and prototypes, which will typically be scale models, in either air or vacuum environments. Accurate test data are needed to validate analytical methods for these structures in one or more of the following three conditions: stationary (static shape), vibrating (modes of vibration), or deploying (deployment dynamics). 
The selected technical focus for making these measurements is close-range photogrammetry, a flexible and robust technology with demonstrated potential for measuring Gossamer-type structures. ${ }^{7-9}$ Static shape measurements are the simplest to make, requiring two or more still photographs of the structure from convergent viewing directions. Vibration measurements are more difficult to obtain, requiring synchronized image sequences from multiple cameras. With vibrating structures, off-line data analysis is simpler than real-time analysis, which needs special hardware and software and can be limited by computational speed to a few simultaneous measurement points. The most difficult situation is quantitative measurement of the unsteady dynamic characteristics of inflating or deploying structures. This case is like vibration measurement, but must also handle large geometry changes and target obstructions that can occur as a function of time.

Industrial photogrammetry systems are available for making highly accurate ( 1 part in 100,000+) structural measurements. ${ }^{10.11}$ However, they were not considered a cost-effective choice for this basic research effort (which includes collaborative research and development in academia and small businesses) because of their high purchase and maintenance costs. To the extent possible, this work uses consumer digitalcamera and software technologies that are affordable to most organizations and provide acceptable accuracy. Occasionally, a Geodetic Services Inc. V-STARS industrial photogrammetry system can be borrowed for measurement comparisons. ${ }^{\dagger}$

The objective of this paper is to document initial experiences at the NASA Langley Research Center using various hardware and software for photogrammetry of Gossamer research structures. The paper has two sections. The first section summarizes experiences with seven laboratory test articles. illustrating some advantages and challenges of imagebased measurement of Gossamer structures. The second section explains the ten main steps of close-range photogrammetry using recent data from a $2-\mathrm{m}$ solar sail model as an example.

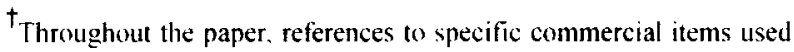
in this research are not an official endorsentent or promotion of any product by NASA or the U nited States government.
}

\section{TEST ARTICLES}

Figure 1 shows seven Gossamer-class test articles measured with photogrammetry at LaRC. The first three are flight prototypes suitable for use in space (Figs. $1 \mathrm{a}-\mathrm{lc}$ ), and the others are generic research structures built for technology development purposes only. The following subsections discuss salient points of each project.

\section{5-m inflatable parabolic reflector}

Figure $1 \mathrm{a}$ is a 5 -m-diameter inflatable parabolic membrane reflector, which weighs only about $4 \mathrm{~kg}$. In space. it can serve as either a microwave antenna or a solar concentrator. The 3D coordinates of 521 attached retroreflective targets were measured with photogrammetry using four Kodak DC290 (2.1megapixel) digital cameras. These cameras had the highest resolution of any consumer model at the time. The test occurred in a closed chamber to minimize air currents. Estimated measurement precisions were $1: 28,000$ ( 1 part in 28,000) in the horizontal direction, $1: 14,000$ in the vertical direction, and 1:5,000 in the camera direction. Later tests showed that measurement precision would improve somewhat by increasing the number of camera locations (from four to nine), their angular separation, or the image resolution. The focal length of a best-fit parabolic surface for the measurements was $3.050 \mathrm{~m}$, which closely correlated with the design focal length of $3.048 \mathrm{~m}$. The root-meansquare deviation from an ideal parabolic shape was about $1.5 \mathrm{~mm}$. Additional details of this work are published elsewhere. ${ }^{12}$

\section{1-m flexible Fresnel lens}

Figure $\mathrm{lb}$ shows test configurations for static-shape (top) and dynamic (bottom) measurements of a $1-\mathrm{m}$ long, membrane solar concentrator. These tests supported development of a proposed space flight experiment. ${ }^{13}$ The test article is a patented, flexible Fresnel lens that refracts light onto a narrow line of solar cells, requiring only $12 \%$ of the cell area of traditional spacecraft solar arrays, which reduces weight and cost. In service, a $3 \times 8-\mathrm{m}$ array would contain 280 of these pop-up lenses. Two cameras were arranged to measure the static shape of the lens with projected circular dots. Although the projected dots had 
good contrast without spraying the lens with a diffuse coating (not permitted in this case), target centroids could not be accurately obtained because of the prisms molded into the membrane. Vitration tests were then conducted using 40 adhesive circular targets and two close-up miniature video cameras for stereo videogrammetry. The bottom of + ig. Ib shows a typical image pair. Image sequencs were successfully processed to obtain $3 \mathrm{D}$ target coordinates versus time. A scanning laser vibrometer (with sub-micron precision) made corroborative vibration measurements for comparison. Factors affecting the achievable photogrammetric accuracy in this application were:

- Marginal target image size at eige of field of view,

- Image intensity variation over iarger targets. and

- Variation in background im zge intensity due to surface reflection and transmis ion characteristics.

\section{15- $m$ inflated, rigidized tube}

Long slender tubes proposel for solar sail support structures and other Gossamer, pacecraft are difficult photogrammetry test objects. Finure Ic shows a 15-mlong, rigidized aluminum-laminate inflatable tube with a length-to-diameter ratio of 100 hanging vertically. The cross-sectional shape and straightness of the tube significantly affect the axial strength and buckling properties, ${ }^{\text {it }}$ so photogrammetric measurements of these quantities were desired. The thinness of the tube wall prevented installation of traditional adhesive targets without risking loc.l damage, so an unconventional measurement approach was designed. A stationary camera on a tall ladde photographed the tube against a dark background as the tube was rotated axially in 30-degree steps. In each photograph, the distances from the edges of the tube to two stationary plumb lines were measured. The se dimensions gave the desired cross-sectional shape and straightness of the tube over most of its length.

\section{0.7-m oscillating Kapton membrane}

The four images in Fig. 1d slow one epoch (instant of time) of a 40-sec, 300-frams video sequence of an oscillating Kapton membrane with 100 illuminated retroreflective targets. A meta! frame tensioned the membrane by its corners into a slightly warped shape, and the frame was suspended by strings. An electrodynamic shaker attached to the bottom of the frame slowly moved the membrane back and forth at $10.0 \mathrm{sec}$ per period. The image sequence captured four periods of the repetitive motion. High membrane tension and slow speed of motion avoided local vibration of the membrane. This dataset, representing the rigid-body motion of the structure, is a good test case for development of new or improved motion analysis software. Accurate photogrammetric analysis of the sequence should show four identical periods of a rigid, warped surface swaying back and forth in the manner described above. The same motion analysis software can then be applied to flexible-body datasets to identify structural dynamic modal parameters (assuming the vibration is large enough to detect with cameras). ${ }^{15}$ Note that both upper images in Fig. Id have a "hot spot" from reflection of a light source located at another camera. It is impossible to measure photogrammetry targets there. The hot spots change location as the membrane oscillates.

\section{3-m hexapod reflector}

Figure le shows a 3-m-diameter, reflective membrane research structure developed by ILC Dover. Inc., Tennessee State University and NASA Langley for active shape and vibration control experiments. It is not an actual spacecraft concept, but contains generic components of proposed inflatable Gossamer observatories. The structure uses a Stewart Platform configuration, also known as a hexapod--a design for controlling all six degrees-of-freedom of the triangular feed platform. On all six tapered tubes holding the feed platform and on the membrane boundary are piezoelectric actuators that can dampen (or create) vibrations. Control experiments will compare various feedback circuits or algorithms to sense and adjust the static shape or dynamics of the system using the actuators to optimize a selected performance objective. Photogrammetry can measure the membrane shape before and during the control experiments. The righthand side of Fig. le shows about 550 dots projected onto the back of the membrane, ${ }^{16}$ which also has a reflective aluminum coating like the front surface. Two cameras, one on either side, photographed the dots. Although the surface is shiny, sufficient photographic contrast was obtained in a darkened room with long image exposure times (about $30 \mathrm{sec}$ ) for accurate measurement of the static shape. 


\section{4.5- $m$ inflatable tripod}

Understanding the deployment dynamics of Gossamer space structures is a key element of making them a reliable and practical technology. Videogrammetry (photogrammetry using image sequences) is a logical way to measure deployment dynamics of Gossamer structures by tracking discrete targets on the structure with multiple cameras. ${ }^{17}$ Figure If shows three stages of inflation of a simple tripod constructed of black, 150-micron-thick, polyethylene tubing. Basic-research investigations used this material because it is inexpensive and rugged enough to withstand many inflation/deflation cycles. Lowpressure air inflated all three legs simultaneously in this experiment. Clearly, a significant issue for reliable videogrammetric tracking of targets on inflating structures is obstruction of targets by folds of the material or by other members. Some successful realtime target tracking occurred in this experiment for up to two of the three legs simultaneously using two synchronized cameras; however, targets were frequently lost from view of either or both cameras. Recording the entire deployment sequence, and then post-processing the images in reserve order, generally is the best approach for obtaining 3D target trajectories in deployment tests. Algorithms for extrapolating paths of targets that move temporarily out of view can improve tracking performance.

\section{Partial 10-m and two complete 2-m solar sail models}

Fig. Ig shows three solar sail structural test articles. ${ }^{18}$ The large one on the left is half of a fourquadrant, $10-\mathrm{m}$ sail concept (the length of each edge is $10 \mathrm{~m}$ ). Those on the right are $2-\mathrm{m}$ scale models of different sail designs. These research structures are in a 16-m-diameter vacuum chamber, large enough to accommodate testing of a complete $10-\mathrm{m}$ solar sail model in both horizontal and vertical orientations. All three structures use aluminized Kapton membranes (25micron-thick) that are shiny, but with sufficient diffuse reflection for 3D photogrammetry. Useful space missions require sail sizes of at least $70 \mathrm{~m}$ with membrane thicknesses of less than 7 microns. The 10-m test article has 80 distributed, 28.5-mm-diameter, retroreflective targets for laser vibrometry and photogrammetry measurements of overall shape and dynamic characteristics. High-density dot projection has also been used on a portion of the four-quadrant, 2 $\mathrm{m}$ sail, shown in the lower-right corner of Fig. $1 \mathrm{~g}$, to measure its static shape with high spatial resolution. Note that the four-quadrant, $2-\mathrm{m}$ sail has four individual triangular membrane sections, tensioned by slender aluminum rods running between them.

\section{PHOTOGRAMMETRY}

Figure 2 is a flowchart of the ten main steps of photogrammetry consistent with PhotoModeler Pro, one of the software programs used in this research. The remainder of the paper discusses each step individually using data from a recent test of the four-quadrant, 2-m solar sail as an illustrative example. Note that the flowchart is a "closed loop" since lessons learned in each application lead to method improvements in later applications.

\section{Step 1: Establish measurement objectives and accuracy requirements}

The seven projects discussed in the preceding section show how photogrammetry can measure a wide variety of structures, using a variety of experimental methods. There are only three top-level measurement objectives for Gossamer structures (static shape, modes of vibration, and deployment dynamics), but there are many ways to estimate each type of data. Establishing specific measurement objectives and accuracy requirements is important for selecting proper test methods.

An important consideration in developing groundtest objectives is a good understanding of mission requirements and important design issues so they can be adequately validated. For example, with solar sails, the two biggest technical concerns (at least in early demonstration experiments) is proper deployment of the sail and controllability. Next is the ability of the sail to accelerate as expected using the momentum exchange imparted by sunlight (photon) reflection. All three aspects relate to one of the types of data that photogrammetry can measure. Specifically, photogrammetry can measure deployment dynamics and modes of vibration, which relate to the deployment and attitude control aspects, respectively. Sail 
acceleration performance in spac: relates directly to the operational shape of the deployed membrane, which can also be determined with photogrammetry.

A good estimate of the required measurement accuracy for each photogrammetry project is also important, avoiding both under- and over-estimating the requirements. Under estimalion can lead to unacceptable or unreliablc measurements. Over estimation can waste time and resources because the cost versus accuracy relationship is one of diminishing returns at higher accuracy levels. Photogrammetric accuracy (specified in parts per thousand of the largest dimension of the structure) can vary by more than three orders of magnitude dependin:- on the method and equipment used, ranging from $1: 1,000,000$ with largeformat film cameras to und $1: 1000$ with lowresolution consumer cameras and manual feature marking in images.

\section{Step 2: Select and calibrate suitable cameras and lenses}

Modern close-range photo:rammetry uses digital cameras almost exclusively rather than traditional film or analog (for video) equipment because of several advantages, including:

1. The images are immediately available for computer analysis (usin removable storage media or cable connection).

2. The photogrammetrist can take many extra pictures at the test site at no additional cost using different camera and lighling settings and select the best images later for the analysis, and

3. The measurement accurac san be higher than for standard 35-mm film, which can shift relative to the camera lens. Also, inage transmissions (for video) are higher quality using digital data lines.

There are also some disadvantages of digital cameras compared with film or analog (for video) equipment, including:

1. Higher prices (at least $3 x$ higher than comparable film cameras), but these are fully recovered by eliminating film and developing costs,

2. Maximum image resolution capability is still achieved by medium- or large-format film cameras designed and calibrated for photogrammetry, and

3. Cable-length limitations of digital video systems, which is typically less than $10 \mathrm{~m}$ without increased noise.

Figure 3 describes two types of digital cameras used in this research. There are at least 200 other models of consumer, scientific, and professional digital cameras on the market, many with similar specifications. Four Olympus E-20 and two Pulnix TM-1020-15 cameras are available at LaRC for staticshape and dynamic measurements of Gossamer structures, respectively. Several other types of cameras are also available for data comparisons.

Accurate photogrammetry requires precise knowledge of the optical characteristics of each camera. referred to as the internal camera parameters. The process of measuring these properties is called camera calibration. 19 At a minimum, the following information is required for each camera: sensor format (pixel size and number of pixels), principal point (intersection of optical axis with the imaging sensor), photogrammetric principal distance (distance from projection center of the lens to the principal point), and lens distortion characteristics (radial, decentering, and possibly others). Note that the photogrammetric principal distance is synonymous with the focal length of the lens when focused at infinity.

The PhotoModeler Pro software contains a simple procedure for computing internal camera parameters by analyzing photographs of a grid of targets projected onto a flat wall. To illustrate the procedure, Fig. 4 shows typical photos of the camera calibration grid. It is a rectangular mosaic of black and white triangles with a coded dot pattern in each corner. The procedure uses six camera locations and eight photographs. Three locations are on the left side and three on the right side of the grid at low, medium, and high elevations. The fourth photograph on each side is at medium elevation with the camera rotated 90 degrees. The user also measures and inputs the distance between the upper-left and lower-right corners of the projected grid. PhotoModeler uses a mostly automated procedure to process the eight photographs. Camera parameters computed by the method are: format aspect ratio, principal point, photogrammetric principal distance. 
two coefficients of radial lens distortion (usually the largest component of lens distortion), and two coefficients of decentering lens distortion (caused by any misalignment in the lens). Note that industrial or other close-range photogrammetry systems may use a more comprehensive calibration procedure with many additional images and non-planar target locations.

\section{Step 3: Select type, size, and distribution of targets}

Photogrammetry achieves the best accuracy using high-contrast, solid-colored circles as targets. Targets can be light-colored on dark background or darkcolored on light background, the former being more common. Targets cover each part of the structure with enough density to define its shape, usually without being placed at specific locations, though there can be advantages to placing some targets at known coordinates. Circular targets appear in photographs as elongated ellipses, with the elongation depending on viewing angle. Accurate calculation of target centers (centroids) for photogrammetry requires both axes of the ellipse to be about five pixels in size or larger.

This research uses three types of solid-colored circular targets: diffuse, retroreflective, and projected. Diffuse materials, such as common white paper, reflect light in all directions. Retroreflective materials, such as highway road signs or markers, reflect light mostly back in the direction of its source, significantly increasing visibility in that direction alone. Projected targets, typically white dots from a standard slide projector, are an attractive alternative for static-shape measurements of delicate Gossamer structures, but are not as useful as attached targets for dynamic measurements because they do not move with the structure. Photogrammetry can measure the 3D shape of a structure at each instant of time with projected dots: however, motion time histories of specific points on the structure cannot be obtained without interpolation or other assumptions.

Figure 5 shows retroreflective and projected circular targets. Figure $5 \mathrm{a}$ is a retroreflector on a black background, with and without the camera flash turned on. Without illumination, the target is dull gray in color. With illumination, it is bright white--many times brighter than a diffuse white surface. If the exposure is optimized for the retroreflective dots in the images, as in Fig. 1d, illuminated retroreflective targets appear as bright white dots on a dark background and are excellent photogrammetric targets that computer software can automatically locate and mark.

Figure $5 \mathrm{~b}$ compares white dots projected onto two different membrane materials. The left-hand side is aluminized Kapton, the same material used for the solar sails in Fig. Ig. It is mainly a specular surface (reflecting light at the same angle as the incident angle). but has a small diffusivity (reflecting light in every direction). The right-hand side is matte Mylar film. which is mainly diffuse. Diffuse materials are much better for photogrammetry since they give more uniform target contrast from different viewing directions relative to the projector. In Fig. 5b. the projector is directly in front of the membranes and the camera is about 30 degrees to the left side.

Membrane materials for proposed Gossamer structures are often reflective or transparent, which are difficult materials to measure with photogrammetry. Special ground test articles may be manufactured with a diffuse white coating on one or both sides of shiny and transparent membranes to simplify photogrammetry with dot projection.

\section{Step 4: Design the photogrammetric geometry and take the photographs}

Designing the photogrammetric geometry (a.k.a., "network design") involves selecting an adequate number and distribution of camera positions. ${ }^{20} \mathrm{~A}$ general guideline is to place cameras at convergent viewing angles, in both the horizontal and vertical directions if possible, at about 70 to 90 degrees angular separation, plus or minus 30 degrees. A key feature of close-range photogrammetry with bundle adjustment is that the camera locations and orientations do not have to be measured, but are calculated by the software along with the desired target coordinates. The bundle adjustment, which is the data reduction procedure preferred by the photogrammetric community, uses an iterative non-linear least squares solution. Although details of this process are beyond the scope of this paper, a brief discussion is provided in Step 8 below.

Each point of interest on the object must appear in 
at least two photographs for 31) determination. although four or more photographs are preferred for improved least-squares accuracy and reliahility. With knowledge of at least one other constraint, such as knowing that all object points lie on a plane, photogrammetry can also use a single camera location. This capability is particularly useful for real-time measurements to reduce computational requirements. Goisamer structure tests will usually use two or more camera locations to compute 3D structural coordinatiss without assumptions or constraints.

Figure 6 shows the equipnent for dot-projection photogrammetry of the 2-m solar sail model (or other small test articles). This is a laged photograph--the actual test configuration was sumewhat different. To minimize hot spots in the imal!es, the projector was moved to the floor and angled up toward the sail at about 45 degrees, causing the main light beam to reflect mostly above the cameras. Forly pictures were shot using eight camera locations in front of the sail (four across at each of two tripod heights) and with five image exposure settings at each lication. With the room darkened, a standard 35-mm slide projector had enough power to project about 1500 dots with adequate contrast on only the lower-right corner ol this shiny membrane. indicated by dashed lined lines in Fig. 6. The size of this area is about $835 \times 585 \mathrm{~mm}$. Theater projectors with higher power are available tor larger structures.

Photogrammetry requires targets with good contrast that are in reasonable focus. Generally, the best way to take the photographs is using a small aperture setting ( $f / 8$ or higher) to obtain guod depth of field (i.e.. the depth in the object that is simultaneously in focus) while minimizing, or even eliminating, focusing requirements. Using a small ap.rture and focusing the camera to optimize depth of ield, it is possible to simultaneously focus all object: in the picture from a short distance in front of the camera out to the horizon. However, using a small aperture requires a slower shutter speed or brighter illuminitlion to obtain adequate image exposure. Tripods should be used with exposure times longer than about $30 \mathrm{~m}$ sec to avoid camera movement. Most consumer digitil cameras have zoom lenses, and it is important to $b_{c}$ : sure that they are set properly. Normally either the minimum or maximum zoom setting (focal length) and infinity focus are used to simplify the process and improve repeatability. Any changes in the zoom or focus settings require new camera calibration data.

Figure 7 shows the best pair of images among the 40 that were taken of the $2-\mathrm{m}$ solar sail. The contrast in these images is higher than observed with the naked eye for this reflective membrane. Contrast enhancement occurred using long camera exposure settings of about $30 \mathrm{sec}$ (i.e., long integration times). The images were shot using the longest focal length of the camera (36 $\mathrm{mm}$ ) so that the photographer stayed as far away from the membrane as possible to avoid causing air currents that would move the sensitive film. For the images in Fig. 7, the cameras were about $3 \mathrm{~m}$ from the structure and separated by about $3.5 \mathrm{~m}$. Maximizing the test article image size in each photo increases accuracy. The projected dots in the left photograph occupy $85 \%$ of the image and in the right photograph. they occupy $74 \%$. A hot spot occurs in the right image from slight twisting of the membrane edge that redirected the main light beam toward the camera. The occurrence of hot spots on shiny materials with dot projection is almost unavoidable.

Photographic images are inherently nondimensional (e.g., one cannot tell from photographs alone if the solar sail is $2 \mathrm{~m}$ or $20 \mathrm{~m}$ in size). For scaling purposes and for initial calculation of camera locations and orientations, ten light-colored adhesive targets were placed on the membrane and are visible in the photos with close examination. The measured distance between a widely separated pair of adhesive targets provided physical scaling for the resulting 3D photogrammetric model.

\section{Step 5: Select data analysis software and import the images}

Close-range photogrammetry traditionally has been a specialized technology with relatively few software developers. Most photogrammetry software is one of the following three types: 1) Part of a turnkey system and interfaces with one particular camera only, 2) Developed and used by an individual or consulting firm and not available for sale, or 3) Research code written at universities and used primarily by its developers and students. However, with rapidly increasing capabilities of digital cameras and personal computers in recent years, some general-purpose photogrammetry software 
has appeared on the market. These products can analyze images from any source. PhotoModeler Pro, one of the consumer software products, has been used successfully throughout this research. The remainder of the paper describes the photogrammetric analysis of the solar sail images in Fig. 7 with PhotoModeler. Other software products provide similar capabilities.

First, the images are transferred from the cameras to the computer. Most digital cameras use removable, solid-state memory cards about the size of a matchbox, available in capacities as high as $512 \mathrm{MB}$. For the Olympus E-20 camera used to take the solar sail pictures, each card holds up to 150 JPEG images at the maximum resolution of $2560 \times 1920$ pixels. The card is removed from the camera, inserted in a peripheral card reader attached to the computer, and the images are transferred just as floppy disk files are copied. The PhotoModeler software is then started, and the images are selected and imported into the program for analysis.

Next, the user associates each image with its specific, previously calibrated camera (the cameras can be entirely different types). This allows the proper internal camera parameters, obtained from calibration, to be used with each image. Traditionally, photogrammetric measurement of stationary objects uses only one roving camera, and there are some accuracy advantages of this approach (by running a self-calibration procedure during the data analysis). However, many Gossamer structures are so flimsy they can change shape from unintentional air currents created by a roving photographer. In addition, tests under vacuum conditions cannot easily use a roving camera. These situations require multiple stationary cameras. Multiple time-synchronized cameras are also necessary for $3 \mathrm{D}$ dynamic measurements.

\section{Step 6: Mark the target locations in each image}

Data analysis begins by marking the locations of the targets in the images. In other words, the $x-y$ coordinates of the centroid of each elliptical target, projected white dots in this case, must be marked as accurately as possible in each image. An important aspect of precision photogrammetry is the availability of subpixel interpolation algorithms that locate the center of solid-colored ellipses to an accuracy of onetenth of a pixel or less. ${ }^{21}$ The $3 \mathrm{D}$ spatial measurement precision obtained with photogrammetry is directly related to this subpixel interpolation factor. For example, the overall three-dimensional measurement precision improves by approximately a factor of two if the center of ellipses is calculated to a precision of $1 / 20$ of a pixel rather than to $1 / 10$ of a pixel.

PhotoModeler contains a robust subpixel marking tool for circular targets. Individual targets are marked by clicking them with the mouse, or all targets in a rectangular region of the image can be selected and marked collectively. The latter approach is called "automatic marking" (or auto-marking) and although it is not entirely automatic, does greatly simplify subpixel target marking in projects with large numbers of points, such as in dot-projection tests.

Auto-marking requires the selection of an appropriate intensity threshold, which is then used to determine the number and location of targets in the images. The software assumes that parts of the image with intensities below the selected threshold contain no targets. The user selects an area of the image to analyze (in this case the entire image is selected), and then interactively moves a slider bar to adjust and select a threshold value. Figure 8 shows various displays that occurred in the left solar sail image as the slider moved from 255 (pure white) down to zero (pure black). The objective is to choose as low a threshold as possible without seeing too much noise in the image or having the targets join together. With dot projection on this shiny membrane, there is uneven illumination so a single threshold value will not work for the entire image. A threshold intensity of 70 was selected as a compromise to get as many correctly marked targets automatically as possible. Note that target marking procedures may be significantly different and more automatic in other photogrammetry systems.

Figure 9 shows the automatically marked points for this image. Most targets were detected and marked with the exception of several in the upper-center of the membrane located in the brightest region and several on the right edge of the membrane located in the darkest region. There are a few other targets in the image that were not found automatically, and they were marked by hand in a second step. PhotoModeler required $70 \mathrm{sec}$ on a $2.2-\mathrm{GHz}$ computer to calculate the subpixel location of 1500 targets using a least-squares matching 
algorithm. Two other marking algorithms are available that are faster but also less accurrte.

\section{Step 7: Identify which points in the images refer to the same physical point}

The second step of the data inalysis is to match the marked points in one image with their corresponding points in the other images. This process is called "referencing" the points. When a point is initially marked on an image, it is assigned a unique identification number. Then. whien a marked point on one image is referenced to a m.trked point on another image, the software reassigns the same identification number to both points indicating they are the same physical location on the structure. In the beginning of the data analysis, the user must perform this referencing operation manually until a certain minimum number of points (at least six) are referenced on all photos. at which time the user "processes" the data. Processing the data runs a photogrammetric bundle adjustment algorithm, described in Step 8

When these calculations firish (typically in a few seconds), the user returns to the Referencing phase. At this point, automatic helper tool: are available to speed up the process. These tools apnear as a result of the initial processing of the data. which yields the spatial location and orientation angles of the cameras. Now. the images are said to be "criented." In a typical PhotoModeler project without control points (special points with known coordinates), the camera locations and orientations calculated above are relative quantities with respect to one of the cameras, usually Camera 1. At this point, it is a good idea to verify that the software positioned the cameras properly. which can be checked easily in the graphical 3D viewer available in PhotoModeler. The viewer show: small camera icons at their locations and orientations relative to targets with calculated 3D coordinates, displayed as small dots. Controls are available to rotate (1) resize the $3 \mathrm{D}$ graphic for better viewing.

Figure 10 illustrates the use of an interactive, referencing helper tool. The user selects one or more points in the first image to reference in the other images. For example, select Point 5820 in Fig. 10a. Once the images are oriented, the software knows the direction of a light ray from Poitt 5820 on the structure to the first camera. It projects this ray onto the remaining images. The photogrammetric term for this projected line is an "epipolar line." The user knows that the desired point should be somewhere along the line. In most cases, this greatly simplifies referencing the point. In Figure 10b, the corresponding target in Image 2 is the only one directly on the epipolar line, located in the third column of points.

PhotoModeler also contains fully automatic referencing algorithms for applications with two or more images (which is standard). These algorithms work best with at least three images, but there is also a new technique for auto-referencing two images of planar or near-planar structures. The constraint provided by the surface shape allows the software to work with the normally ambiguous case of two photographs. The algorithm asks the user to select three or more points with $3 \mathrm{D}$ coordinates that define the nearplanar surface. The software calculates the equation of this plane, then automatically searches for and references pairs of points using the epipolar line and a user-specified distance from the indicated plane. It is difficult to see in the images, but the measurement region in the $2-\mathrm{m}$ solar sail test is, in fact, not planar enough for this algorithm to reference the entire image simultaneously. As will be clear later, the right-hand sail quadrant is significantly displaced outward at the top--by more than $5 \mathrm{~cm}$--from the lower quadrant. This geometry required auto-referencing to be performed in two steps, a separate operation for each region.

Notice in Fig. 10 that many small, bright spots appear throughout the images. The Kapton membrane for this solar sail model is perforated, and the bright spots are from light reflected by the edges of the holes. These spots cause small errors in the calculated centroids of the targets, but the effects are minimal.

\section{Step 8: Process, scale, and rotate the data}

The third and final step of the data analysis is to "process" the data using the bundle adjustment algorithm. In the technical literature, several variations of the bundle adjustment method appear, with different user options and levels of sophistication..$^{22}$ As discussed in the preceding section, the data are processed initially after referencing at least six points in each image. This orients the images. Then the user returns to referencing 
(and marking, if necessary) additional points. It is often better not to reference all remaining points at this time, but stop after adding some additional points and reprocess the data. With the 2-m solar sail images, points were referenced and processed mostly in two large sets (the lower-left and upper-right regions). Then some additional points were added that did not auto-mark or auto-reference previously, such as on the curved edges. This approach avoids wasting time if for some reason the algorithms fail to handle a large number of additional points, usually because of referencing errors. Referencing errors can be located and fixed more easily if a limited number of new points are added at each step of the procedure.

The bundle adjustment algorithm does two things simultaneously: 1) Computes the spatial locations and orientation angles of each camera, and 2) Computes the $3 \mathrm{D}$ coordinates of all referenced points and estimates their measurement precision. Bundle adjustment is always an iterative solution (since the underlying math is non-linear), and hence the calculations continue until a specified consistency or maximum number of iterations occurs. If the object points are distributed on the structure and the photographs are at suitable angles, the bundle adjustment will usually run successfully. In many cases, camera self-calibration or field-calibration are added to the bundle adjustment equations during the data processing to improve the internal consistency of the solution and the accuracy of the point coordinates.

These steps were followed with the two photographs of the 2-m solar sail, and the coordinates of all targets were successfully determined using successive bundle adjustment calculations. The result was 1449 photogrammetrically computed 3D points describing the static shape of the structure. Following each bundle adjustment, the camera locations and orientations were displayed in the PhotoModeler 3D Viewer. The cameras always appeared to be in their proper positions and orientations, adding confidence that the software was working properly. Recall that the software computes the camera positions and orientations from the images.

At this point, the set of 3D points (a.k.a., the "point cloud") can be scaled to physical units and translated and rotated to any desired coordinate system. Scaling and changing coordinate systems in PhotoModeler is a simple matter of selecting two distant points in an image, entering their separation distance in engineering units, and then selecting three points to define the coordinate system. (The three points specify the new origin, direction of a designated axis, and the plane of another designated axis.) In this test, two of the ten small adhesive dots placed on the membrane were used for scaling. and three of the projected dots defined the coordinate system. In some photogrammetry projects, these scaling and coordinate system points may be located off the structure, e.g., on a rigid frame or other stationary support structure.

\section{Step 9: Examine results and export for additional analyses}

Figure 11 shows two views of the final structural model displayed as a point cloud in the 3D Viewer. Note that it was impossible to mark a region of targets at the hot spot in the right-hand image, so the resulting $3 \mathrm{D}$ model contains a hole at this location. There is also a gap between the two individual membrane sections because projected dots on the slender aluminum tube between the membranes were larger than the tube diameter and therefore the centroids could not be accurately calculated.

It is easier to see the shape of the membrane by examining cross-sectional slices through the model at various elevations, shown in Fig. 12. These contours were created by exporting the 3D data from PhotoModeler in ASCII format and doing cubic-spline curve fits to the data points on five horizontal rows of dots. Note that this plot uses different scales on the $x$ and $y$ axes, so the out-of-plane membrane shape ( $Z$ direction) is amplified in the plot by about $20 \mathrm{x}$ relative to the horizontal dimension ( $\mathrm{X}$ direction). The data show a significant displacement of the upper region relative to the lower region by up to $6 \mathrm{~cm}$. This warped shape, caused by the two upper rods of the sail bending considerably forward by gravity, was the initial configuration of the structure. Later (for the picture in Fig. 6), the upper rods were pulled back and tied in a straighter position by cords. Curling of the membrane edges is also apparent in Fig. 12.

Photogrammetric precision achieved in the project can also be examined using the exported data. Figure 13 shows two principal parameters. largest marking 
residual and tightness, that can be studied. The plots show the results for the $14493 \mathrm{D}$ points sorted in descending order. Marking re: iduals are the leastsquares error distances in the camera image planes. Residuals under 1.0 pixel indicate sub-pixel measurement precision. In this application, half of the points had residuals less than $0 . .1)$ pixel, which is good considering the shiny nature of the membranes and suboptimal target contrast. Points with the largest residuals were manually marked points located on the twisted membrane edges.

The second plot in lig. 13 shows the photogrammetric tightness, which measures the maximum distance (as a percentitge of the object size) between any pair of projected light rays from the images to the object point. Due to measurement errors. light rays extending from marked points in separate images to the same object point in space never intersect. The closeness, or tightness, of the intersection is another indication of measurenent precision. In this application, half of the points had tightness less than $0.016 \%$, equivalent to 1 part in 6250 .

\section{Step 10: Lessons learned and how to improve the methods?}

Some lessons learned in this 2-m solar sail photogrammetry application using projected dots as targets that can benefit later projects are:

1. As expected, shiny membranes are difficult, though not impossible, to measure with dot projection. If possible, future solar sai ground test articles should use a diffuse white membrane coating (on one side is adequate), which would simplify and improve shape measurements with dot projection.

2. Higher-power projectors $w$ ith a variety of lenses are needed to measure complete solar sails of 2-m in size and larger at various projection distances and angles.

3. A convenient way to muve the projector to different locations was no available in the test. With a shiny membrane, finding the projector location and direction that minimizes hot spots in the images is helpful.

4. Lightly tensioned membran sove easily from air currents generated by walhing near them. Using multiple simultaneous caneras is preferable to using a single roving camera to avoid this problem. Multiple cameras fired remotely from outside a closed chamber are best to completely avoid unintentional air currents.

5. The use of three or more images from convergent viewing directions simplifies target referencing compared with the use of only two images, particularly for non-planar surfaces. An effective camera network for a square solar sail test would consist of one camera in each corner pointing toward the hub of the sail.

\section{CONCLUSIONS}

This paper summarized experiences at NASA Langley Research Center during the past three years to develop or adapt photogrammetry methods for Gossamer-type spacecraft and components. The research used mainly off-the-shelf digital-camera and software technologies that are affordable to most organizations and provide acceptable accuracy. The first part of the paper discussed seven successful applications on a variety of research structures. The second part discussed the ten main steps of photogrammetry (consistent with the PhotoModeler Pro commercial software program) using data from a recent 2-m solar sail test with projected dots as an example. Solar sails require highly reflective membranes for their operation in space (they are propelled by reflecting sunlight), but shiny membranes are difficult test objects because photogrammetry uses the diffuse component of reflected light, not the specular component. The static shape of the 2-m solar sail was successfully determined. but required long image exposure times of about $30 \mathrm{sec}$. Using a diffuse white coating on future test articles can simplify shape measurements. Many other lessons were learned in these initial applications that will improve future photogrammetry projects with Gossamer structures.

\section{ACKNOWLEDGMENTS}

The authors acknowledge the contributions and support of Alpheus Burner, Paul Danehy, Mark Hutchinson, and Keith Belvin of NASA LaRC. Vaughn Behun of Swales Aerospace, Joe Blandino of James Madison University, Gary Robertson of ShapeQuest, Inc., and former students Louis Giersch and Jessica Quagliaroli, in the development of photogrammetry 
methods for Gossamer spacecraft structures.

\section{REFERENCES}

${ }^{1}$ Mikhail, E. M., Bethel, J. S., and McGlone, J. C., Introduction to Modern Photogrammetry, John Wiley \& Sons, New York, NY, 2001.

'Atkinson, K. B. (editor), Close Range Photogrammetry and Machine Vision, Whittles Publishing, Scotland, UK, 200l.

"Shortis, M. R. and Beyer, H. A., "Sensor Technology for Digital Photogrammetry and Machine Vision," ibid., pp. 106-155.

${ }^{4}$ Cooper, M. A. R. and Robson, S., "Theory of Close Range Photogrammetry," ibid., pp. 9-51.

${ }^{5}$ El-Hakim, S. F. (editor), Videometrics and Optical Methods for $3 D$ Shape Measurement, SPIE Proceedings No. 4309 , Jan. 2001 .

'Jenkins. C. H. M. (editor). Gossamer Spacecraft: Membrane and Inflatable Structures Technology for Space Applications. Vol. 191. Progress in Astronautics and Aeronautics, AIAA, Reston, VA, 2001.

${ }^{7}$ L'Garde, Inc., "Photogrammetry Capability," http:/www.Igarde.com/capabilities/photo.html.

${ }^{8}$ Giersch, L. R., "Pathfinder Photogrammetry Research for Ultra-Lightweight and Inflatable Space Structures," M.S. Thesis, George Washington University, Aug. 2001 (also published as NASA CR-2001-211244, Nov. 2001).

${ }^{9}$ Dharamsi, U. K., Evanchik, D. M.. and Blandino, J. R., "Comparing Photogrammetry with a Conventional Displacement Measurement Technique on a Square Kapton Membrane," AIAA paper 2002-1258, April 2002.

${ }^{10}$ Geodetic Services, Inc., Melbourne, FL, V-STARS Industrial Photogrammetry Systems, http://Www.geodetic.com.

"Imetric SA, Porrentruy, Switzerland, 3D Image Metrology Systems, http://www.imetric.com.

${ }^{12}$ Pappa, R. S., Giersch, L. R., and Quagliaroli, J. M., "Photogrammetry of a 5-m Inflatable Space Antenna With Consumer Digital Cameras," Experimental Techniques, July/Aug. 2001, pp. 21-29.

${ }^{13}$ Pappa, R. S., Woods-Vedeler, J. A., and Jones, T. W., "In-Space Structural Validation Plan for a Stretched-
Lens Solar Array Flight Experiment," Proceedings of the 20th International Modal Analysis Conference, Feb. 2002, pp. 461-471.

${ }^{14}$ Watson, J. J., "Static-Test Results for the Characterization of Inflatable Rigidizable Columns," AIAA Paper 2001-1269, April 2001.

${ }^{15}$ Pappa, R. S., Lassiter, J. O., and Ross, B. P.. "Structural Dynamics Experimental Activities in UltraLightweight and Inflatable Space Structures," AIAA Paper 2001-1263, April 2001.

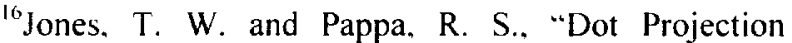
Photogrammetric Technique for Shape Measurements of Aerospace Test Articles," AlAA Paper 2002-0532, Jan. 2002.

${ }^{17}$ Shortis, M. R. and Snow, W. L., "Videometric Tracking of Wind Tunnel Aerospace Models at NASA Langley Research Center," The Photogrammetric Record, Vol. 15, No. 85, 1997, pp. 673-689.

${ }^{18}$ Slade, K. N., Belvin, W. K., and Behun, V., "Solar Sail Loads, Dynamics, and Membrane Studies," AIAA paper 2002-1265, April 2002.

${ }^{19}$ Fryer, J. G., "Camera Calibration," Close Range Photogrammetry and Machine Vision, edited by K. B. Atkinson, Whittles Publishing, Scotland, UK, 2001, pp. 156-179.

${ }^{20}$ Fraser, C. S., "Network Design," ibid., pp. 256-281.

${ }^{21}$ West, G. A. W. and Clarke, T. A., "A Survey and Examination of Subpixel Measurement Techniques," SPIE Proceedings No. 1395, Sept. 1990, pp. 456-463.

${ }^{22}$ Granshaw, S. I., "Bundle Adjustment Methods in Engineering Photogrammetry," Photogrammetric Record, Vol. 10, No. 56, 1980, pp. 181-207. 


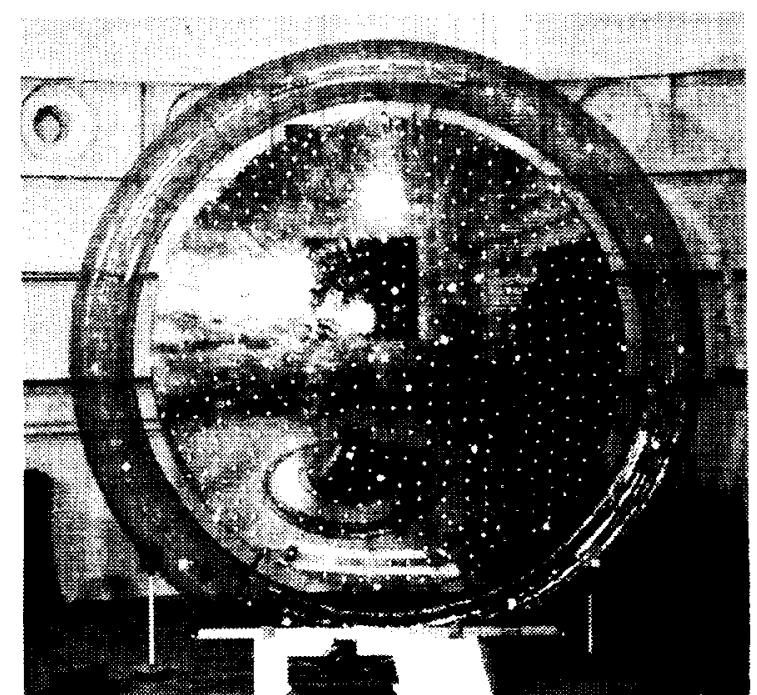

a) 5-m inflatable parabolic reflector
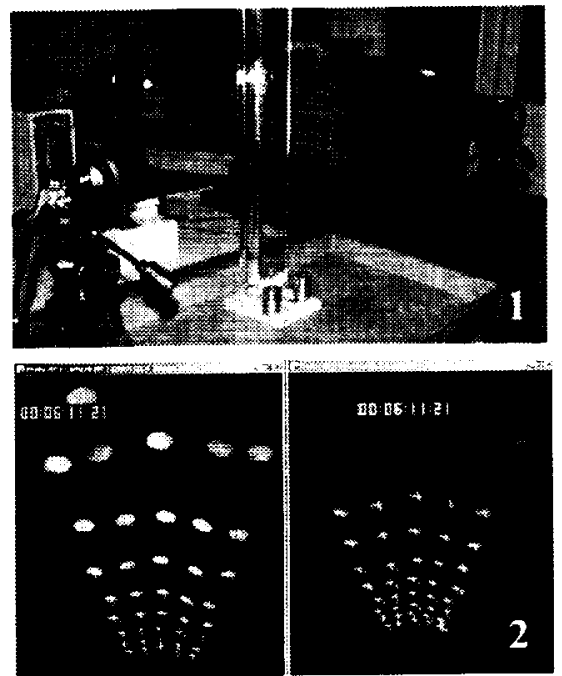

b) 1-m flexible Fresnel lens

(1-projected dots, 2-adhesive targets)

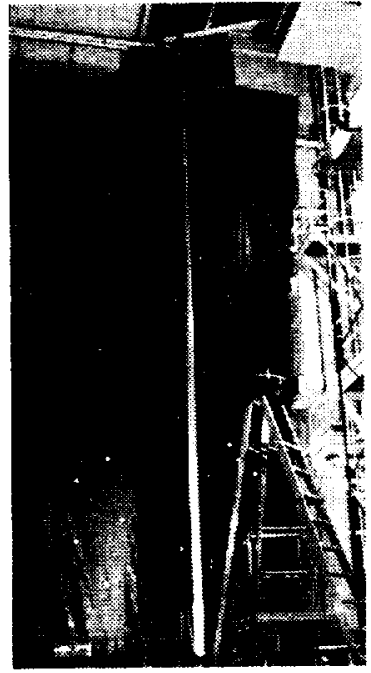

c) 15-m inflated, rigidized tube
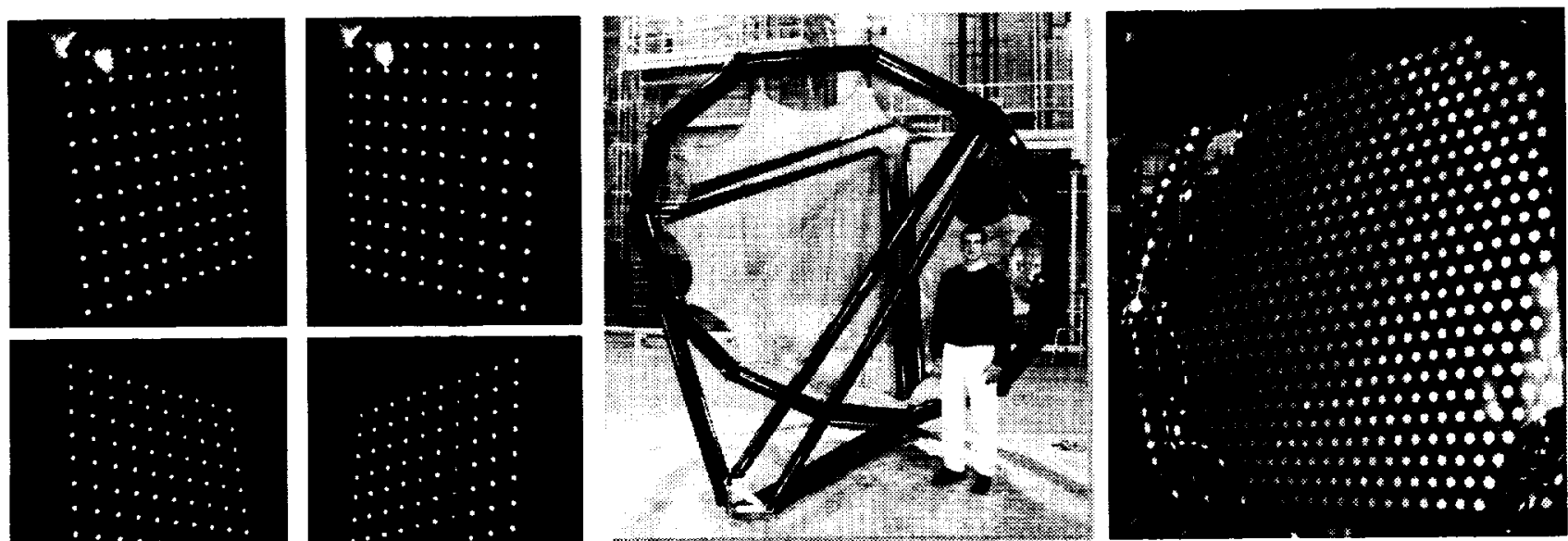

e) 3-m hexapod reflector for active control experiments (projected dots on stretched membrane reflector)

d) 0.7-m oscillating Kapton membrane with retroreflective target.

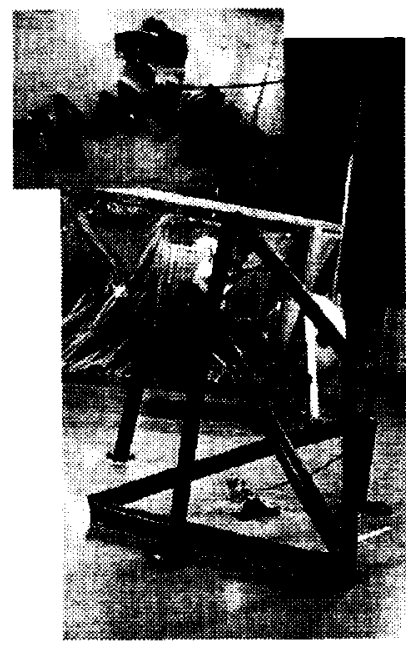

f) 4.5-m inflatable tripod

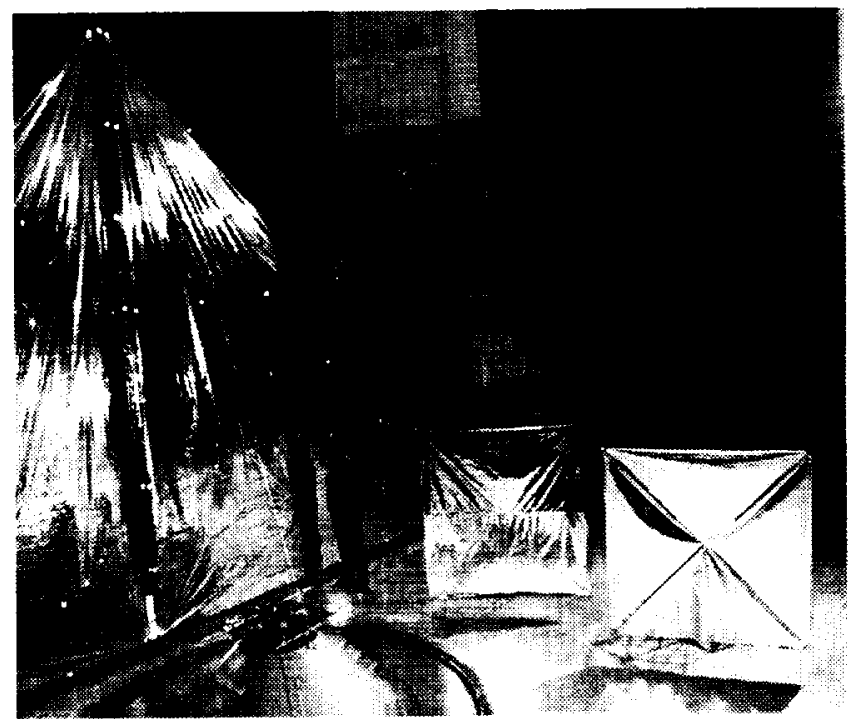

g) Partial 10-m and two complete 2-m solar sail models

Fig. 1. Gossamer test articles.

13

American Institute of Aeronautics and Astronautics 


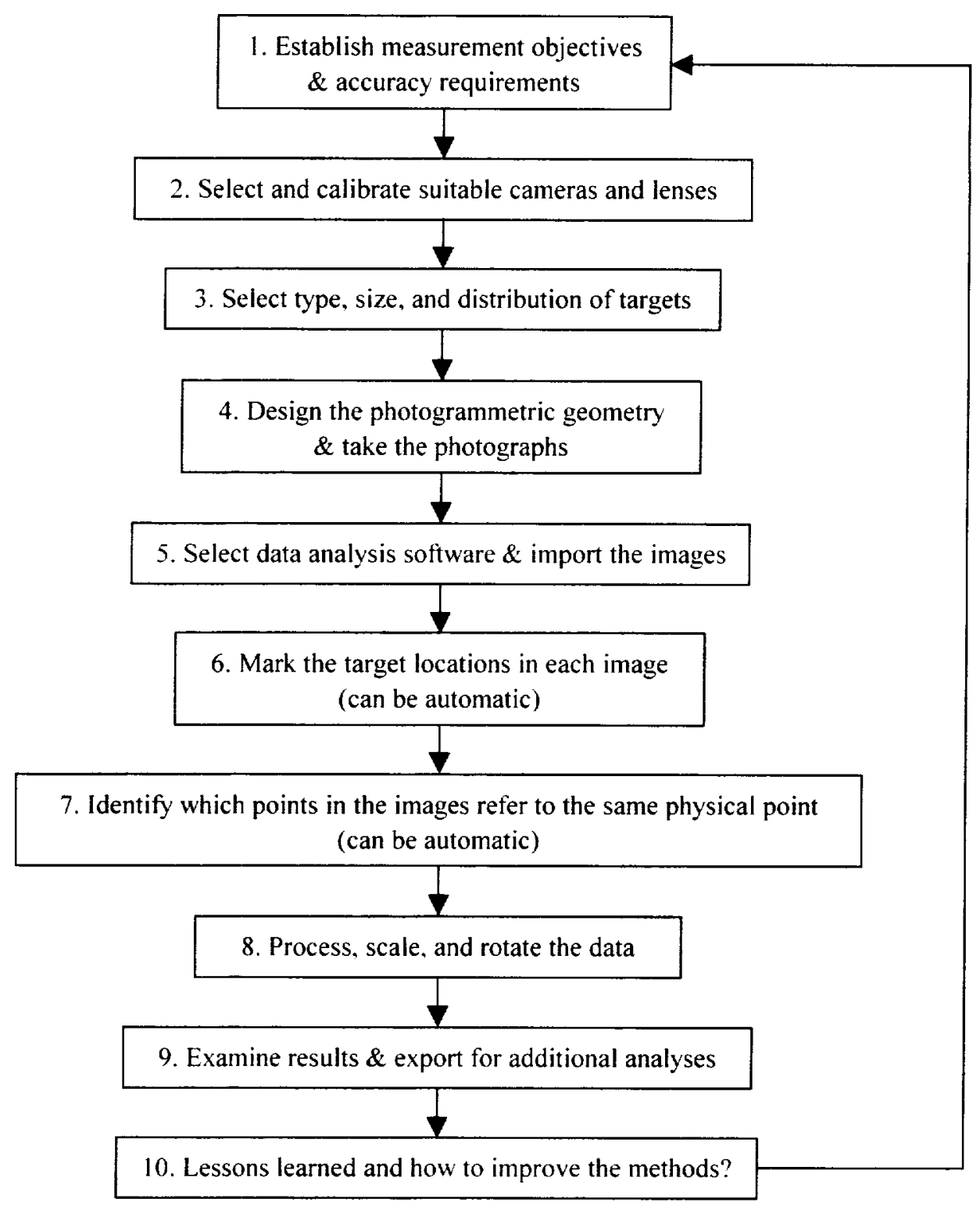

Fig. 2. The 10 steps of photogrammetry.

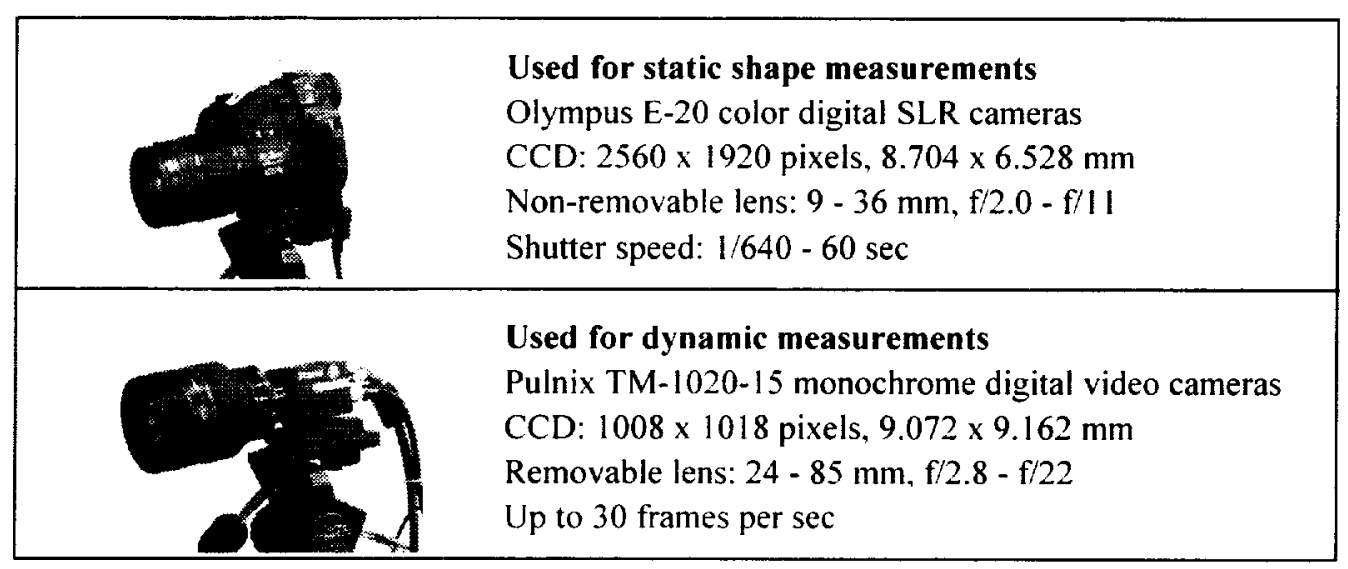

Fig. 3. Camera characteristics. 

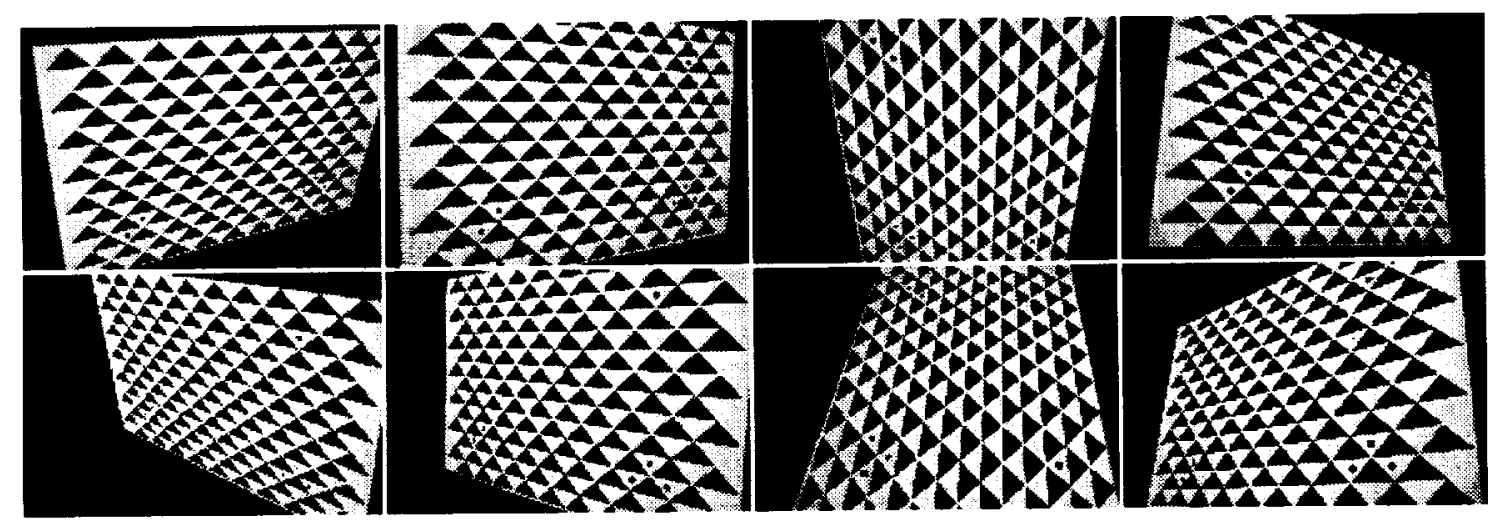

Fig. 4. Camera calibration images.

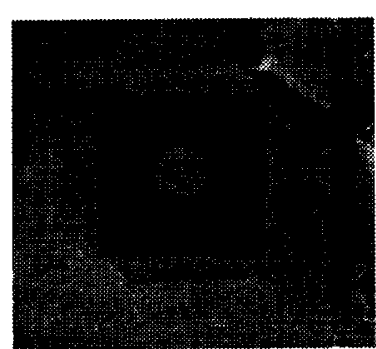

With camera flash off

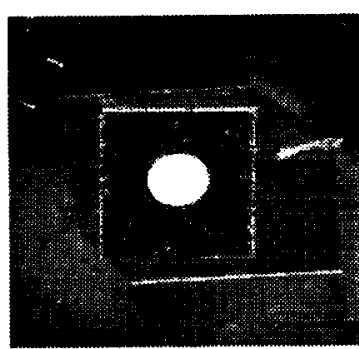

With camera

flash on

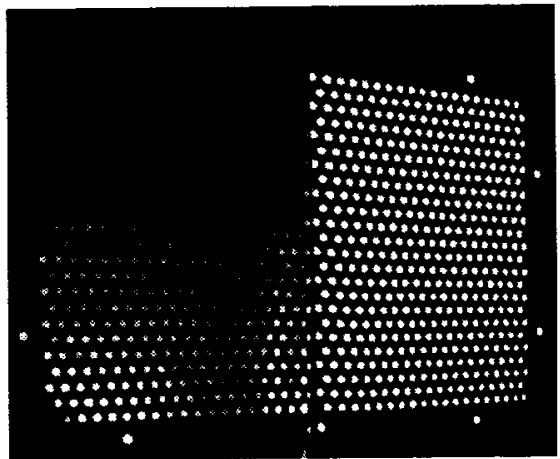

a) Retroreflectors appear bright white when illuminate $\mathrm{J}$ from camera position b) Comparison of projected dots on shiny (left) and diffuse-white (right) membranes

I.ig. 5. Retroreflective and projected circular targets.

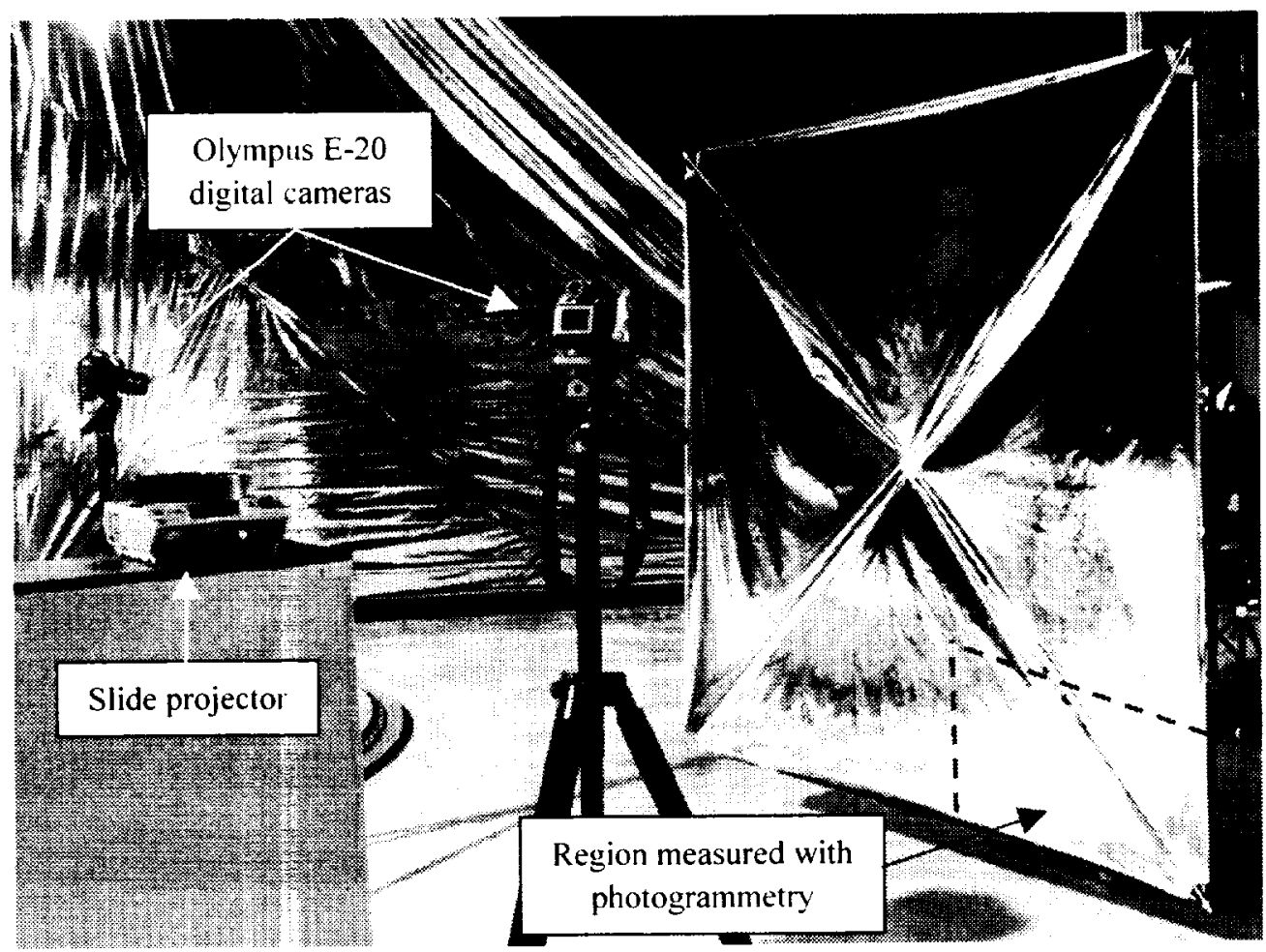

Fig. 6. Equipm .nt for photogrammetry of $2-\mathrm{m}$ solar sail model using projected dots. 


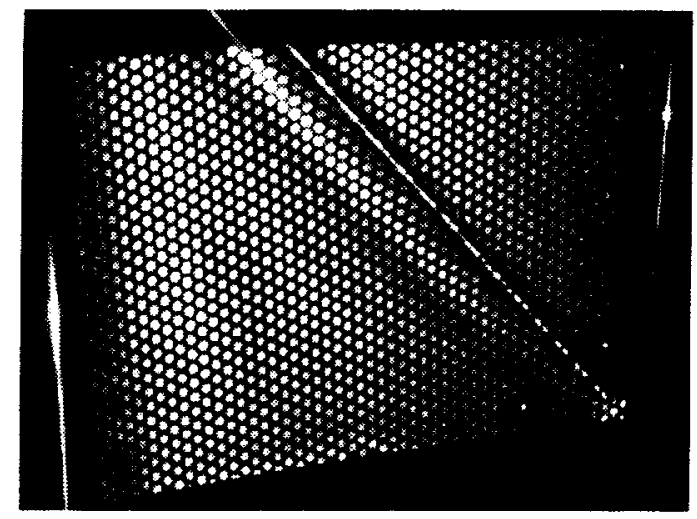

Left image

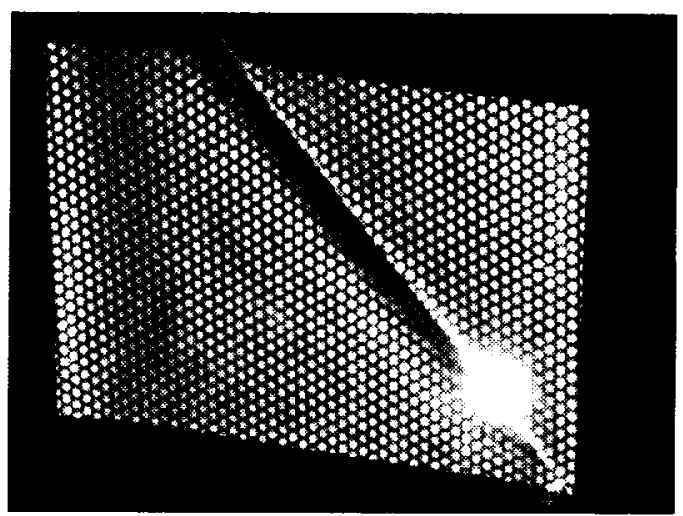

Right image

Fig. 7. Best images of lower-right corner $(835 \times 585 \mathrm{~mm})$ of $2-\mathrm{m}$ solar sail model
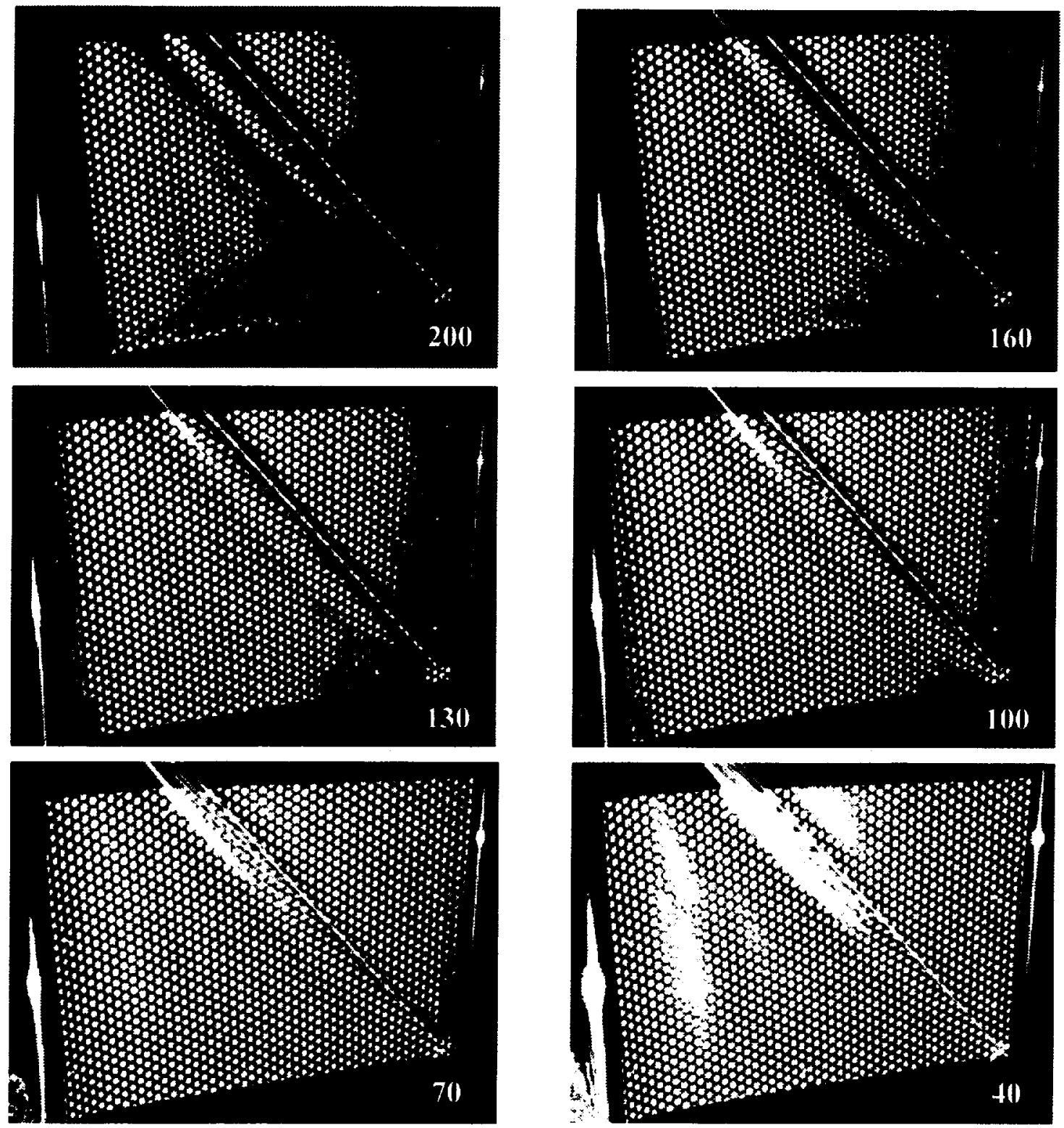

Fig. 8. Left image displayed at various binary intensity thresholds. 


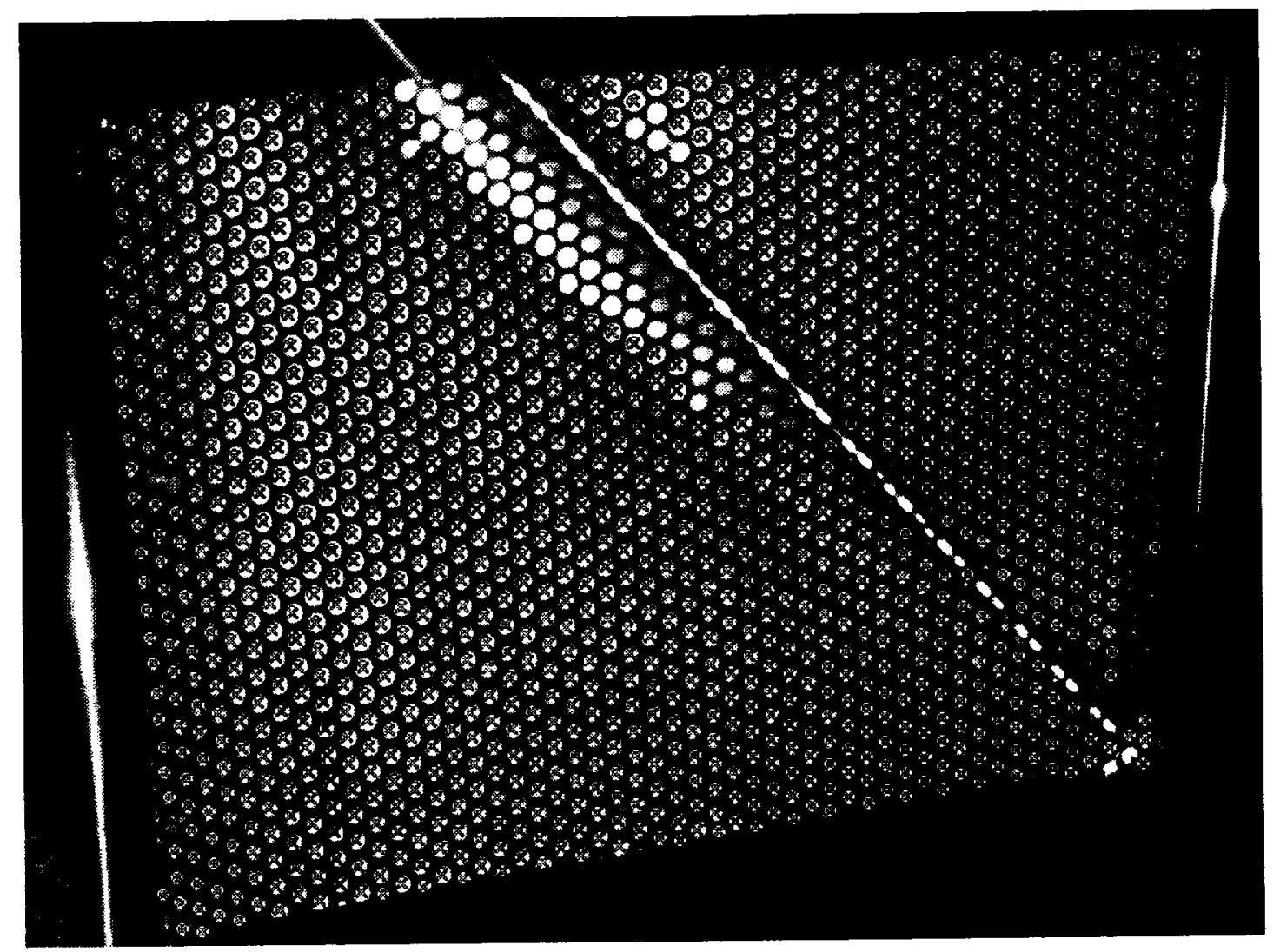

Fig. 9. Automatically marked points using an intensity threshold of 70 .

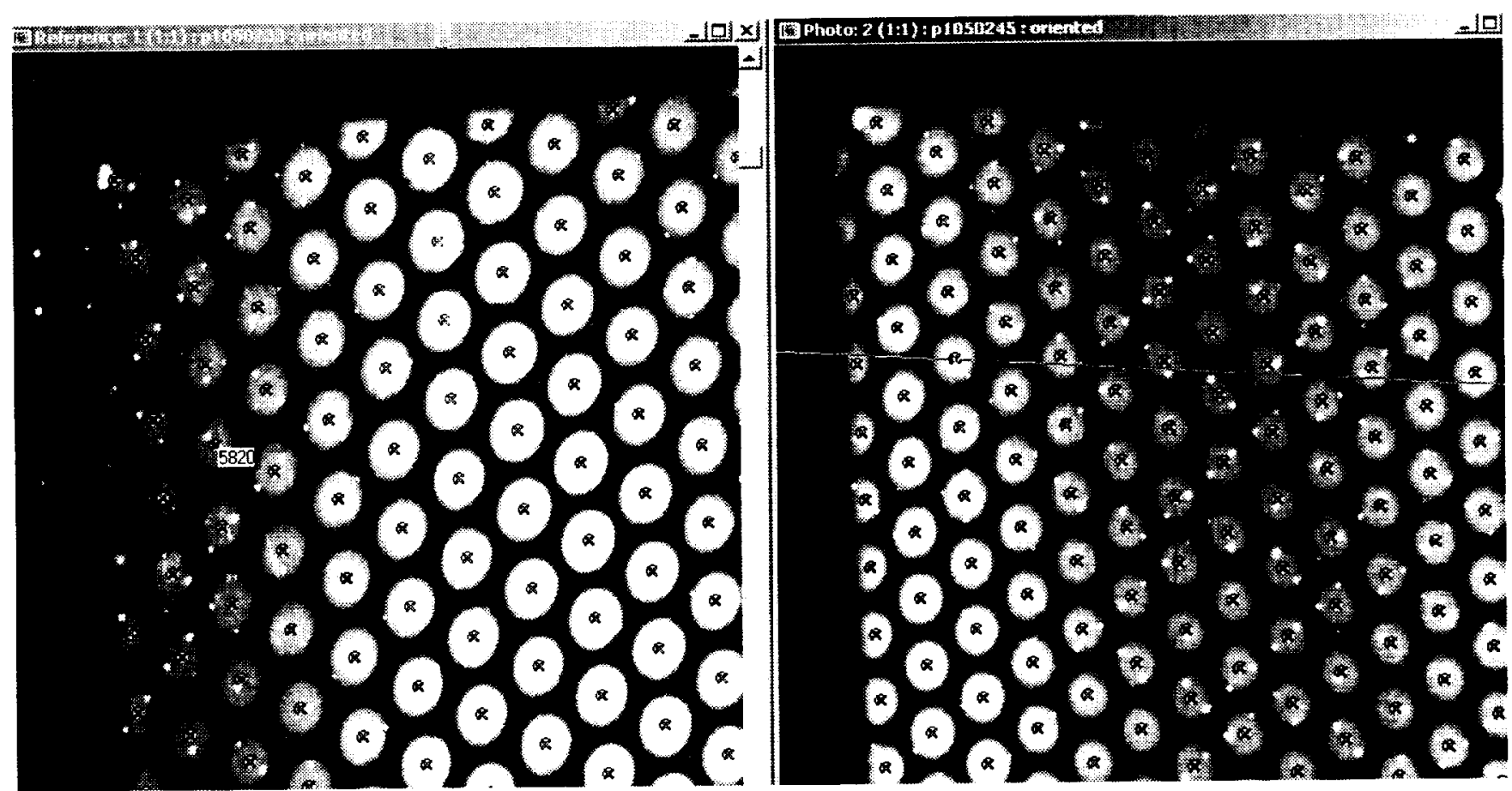

a) Select a point in Image ! (e.g., \$5820)

b) Corresponding point in Image 2 is on the epipolar line

Fig. 10. Epipolar line assists target referencing. 

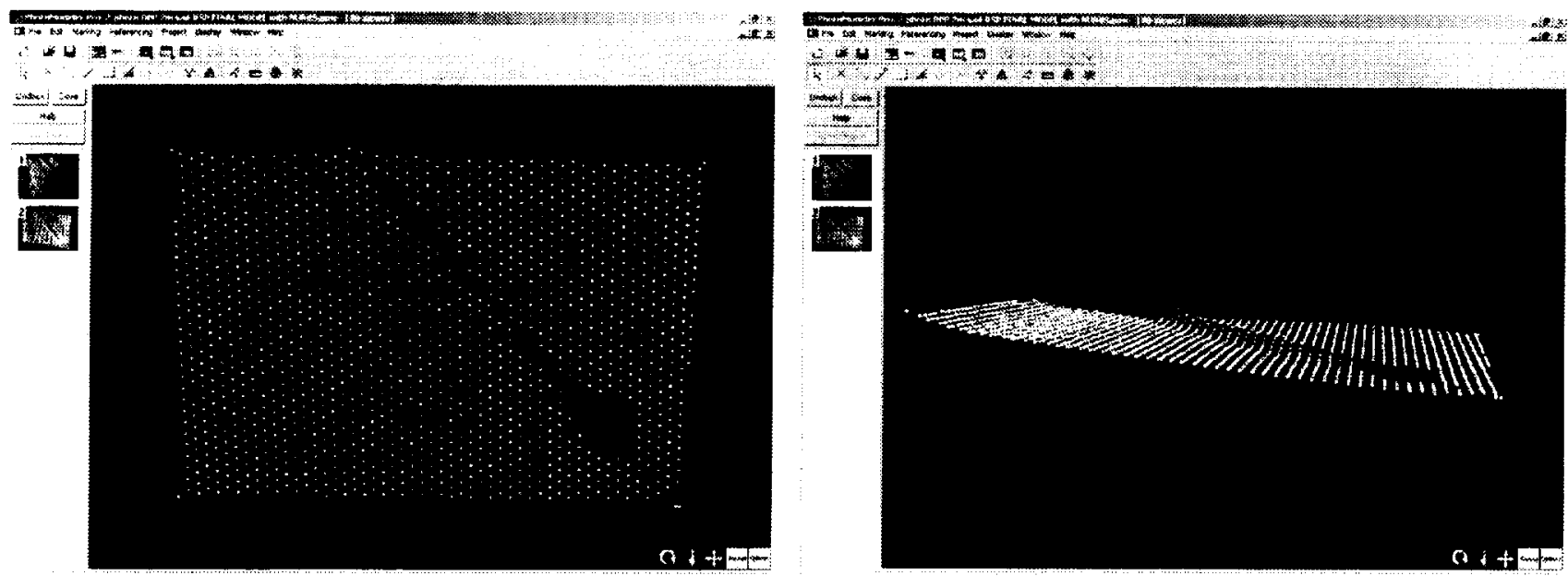

Fig. 11. Two views of 3D point model (1449 points).

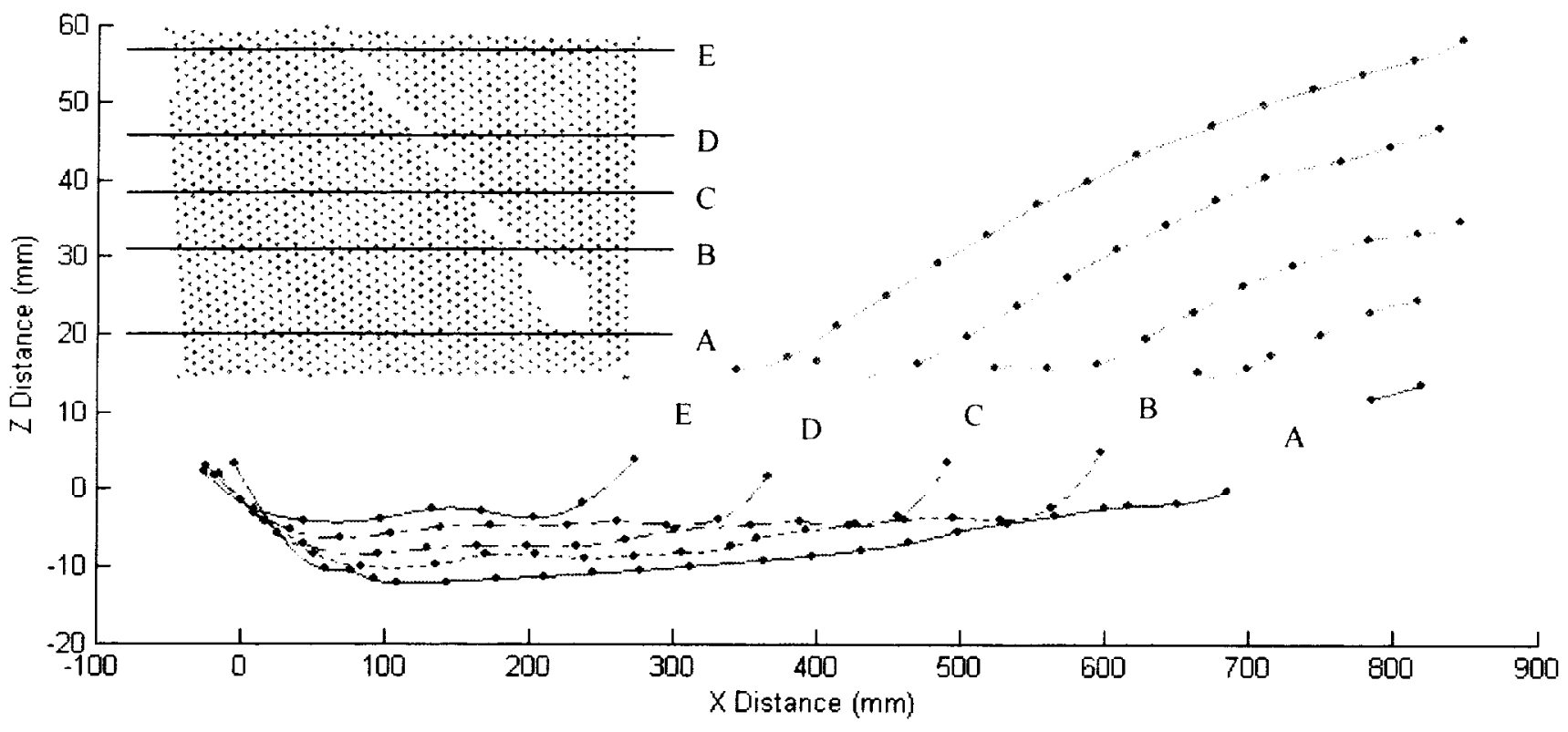

Fig. 12. Out-of-plane membrane shape at various elevations.
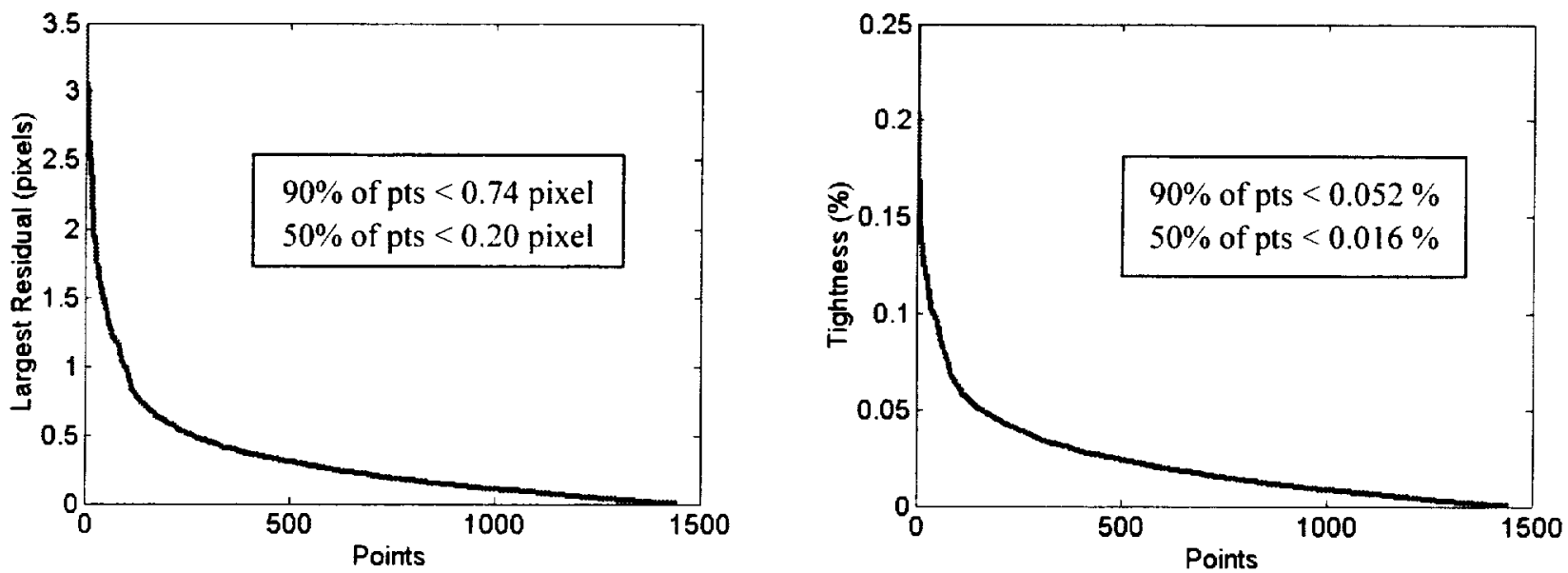

Fig. 13. Distribution of largest marking residuals and tightness (descending order). 